\title{
Effect of thermal lensing and micrometric degraded regions on the catastrophic optical damage process of high-power laser diodes
}

\author{
JoSe LUIS PURA, ${ }^{1, *}$ Jorge SOUTO, ${ }^{1}$ JuAN JimÉneZ ${ }^{1}$ \\ ${ }^{1}$ GdS Optronlab, Dpt. Física de la Materia Condensada, Ed. LUCIA Universidad de Valladolid, Paseo de Belén 19, 47011 Valladolid, Spain \\ *Corresponding author: jlpura@fmc.uva.es
}

Received 28 January 2020; revised 18 February, 2020; accepted 18 February 2020; published 17 March 2020

\begin{abstract}
Catastrophic Optical damage (COD) is one of the processes limiting the lifetime of high-power laser diodes. The understanding of this degradation phenomenon is critical to improve the laser power and lifetime for practical applications. In this paper we analyze the defect propagation inside the cavity of quantum well $(Q W)$ highpower laser diodes presenting COD. For this, we studied the effect of highly localized thermal gradients and degraded regions on the laser field distribution. Finite element method (FEM) simulations are compared to experimental cathodoluminescence (CL) measurements. The presence of micrometric hot spots inside the $Q W$ induces the thermal lensing of the laser field. The laser self-focusing inside the cavity eventually generates a new hot spot, and, in a repetitive way, a sequence of hot spots would be created. This would account for the propagation of the dark line defects (DLDs) characteristic of this degradation mode.
\end{abstract}

http://dx.doi.org/10.1364/OL.99.099999

\section{Introduction}

Laser diode reliability is a field of primary interest for laser manufacturers. One of the key factors limiting high power operation of laser diodes is the catastrophic optical damage (COD). The study of this degradation mode is critical in order to improve the laser power and lifetime as required by different applications, e.g., telecommunications, solid state laser pumping, and medical appliances, among others [1,2]. Understanding the physical mechanisms driving the COD should provide a step forward to increase the laser power and lifetime.

In spite of the many efforts carried out to unravel the details of the COD $[3,4]$, there are still many uncertainties about how it starts, how it propagates, and what are the driving forces leading to COD. COD consists of the sudden quenching of the laser diode optical power after many hours of regular operation. Generally, it is associated with the fast heating of the device near its front mirror facet. This local overheating starts a thermal runaway process leading to the destruction of the laser cavity. The inspection of devices that have undergone COD reveals the presence of defects along the cavity, which give a dark contrast in luminescence mapping techniques, e.g. cathodoluminescence (CL), microphotoluminescence $(\mu \mathrm{PL})$. The typical products of the COD are elongated defects aligned with the laser cavity, the so-called dark line defects (DLDs) [5,6]. The propagation of DLDs is driven by the optical field as they follow the optical path instead of a specific crystallographic direction.

One can establish a general scenario for the COD; it is triggered by the formation of a local heat source in the active region of the laser, generally close to the front mirror, but also internal cavity COD has been reported to occur [7], especially under pulsed operation [8]. As a consequence of the local heating the transparency is lost and the laser radiation can be locally absorbed, resulting in a further increase of the local temperature. Once the thermal stresses induced by the local temperature increase reach the material yield strength a sudden massive formation of dislocations takes place [9]. The presence of dislocations reduces the thermal conductivity [10], leading to an abrupt increment of the local temperature. This mechanism can be associated with the microexplosion suggested by Eliseev [11], or the thermal flashes imaged by Hempel et al using a thermocamera [12]. In previous works, we have set-up a thermomechanical model accounting for COD [13-15]. This model has been further refined [16,17], by focusing on the role played by the nanoscale size of the active layers of the laser [18] and different types of laser structures [19,20].

The propagation of the DLDs, however, has not comparatively received such a wide attention [8]. The defects propagate along the laser cavity, and one of the main questions not yet satisfactorily answered is how this occurs. This is the subject of this work, in which we present an analysis of the effect of highly localized thermal gradients and micrometric degraded regions on the laser optical field distribution. As it is well known, the COD is tightly related to the local optical power density and, therefore, its local enhancement might assist the propagation of the DLDs. 
COD signature in QW lasers

A typical QW laser structure is shown in Fig. 1a. The light generated in the $Q W$ is guided by the two guide layers and confined by the surrounding cladding layers. The pristine guide and cladding layers are transparent to the laser radiation; therefore, the absorption medium must be the QW itself, more specifically, laser self-absorption should initially occur in microscopic overheated regions located inside the QW during operation. As demonstrated by Hempel et al, in pulsed current aging tests the COD starts as a local thermal flash $[8,12]$. Then, in subsequent pulses successive thermal flashes are observed, resulting in a quasi-continuous DLD along the laser cavity. This can be observed in the shape of the DLDs revealed by CL in a $980 \mathrm{~nm}$ InGaAs QW single mode laser aged by current steps, Fig. 1b. A sequence of dark defects that spread slightly out of the QW can be observed. This image matches the observation of sequential thermal flashes separated a few microns from each other. It also suggests that once a COD event takes place and generates one of these dark spots, new events appear sequentially along the QW. A critical point of the COD concerns the propagation of defects, since the laser life ends either once the group of defects has acquired a sufficient size reducing the gain below the threshold value; or, alternatively, when the integrity of the optical cavity is seriously compromised. There is consensus about the role of the laser self-absorption in locally heated regions of the $Q W$, however, one needs to understand how the defects propagate.

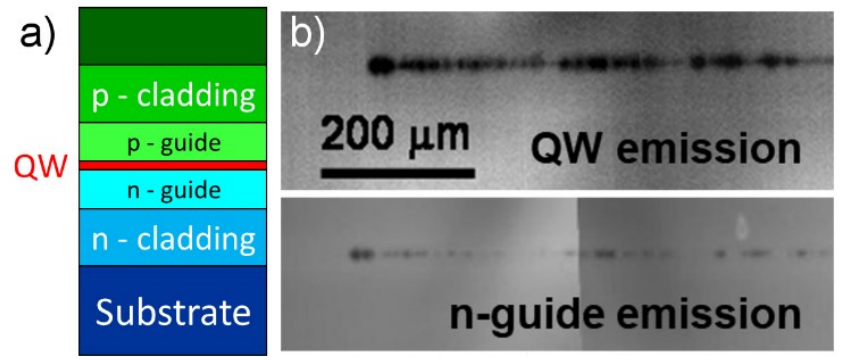

Fig. 1. a) Scheme of a typical QW structure in a laser diode. The light generated in the QW conforms a laser beam along the guide layers by light confinement between the two cladding layers. b) Top view CL images of a degraded laser diode, showing the CL emissions of both the QW and n-guide respectively. The dark lines are aligned along the ridge. The DLDs are localized in the QW (horizontal dark vertical line). The DLD appears discontinuous along the ridge, with a lateral spread in the most degraded points, for which the guide layer also presents dark contrast revealing the degradation of the guide layer in those points

There are two important factors affecting the thermal flashes and the DLDs propagation. On one side, the local enhancement in the absorption coefficient, which is governed by the band gap shrinkage induced by a local temperature increase, while the rest of the active zone remains "cold" and operating. On the other side, the spatial concentration of laser power as a result of the redistribution of the optical field by the presence of regions either overheated or degraded by the laser selfabsorption.

Aiming to understand the DLDs propagation, we have studied the laser radiation field inside the $Q W$ in the presence of micrometric features. We carried out Finite Element Methods
(FEM) simulations in 2D, in order to study two different aspects of the problem: the effect of high thermal gradients and the impact of micrometric degraded regions of material on the laser electromagnetic field distribution.

Modelling

The simulations were carried out by FEM using the commercial software COMSOL Multiphysics. The model considers a $1.42 \mathrm{eV}(870 \mathrm{~nm})$ monochromatic plane wave traveling through a GaAs QW, that reaches a section with a highly localized heat source and/or a locally degraded region of material, simulating a local COD event. Two different effects on the optical field will be considered: the thermal lensing due to the local temperature gradient generated around the hot spot where the COD is launched, and the modification of the optical field induced by the presence of a degraded region in the $Q W$ with modified optical properties.

The effect of the temperature gradient on the laser beam can be described in terms of the thermally induced changes in the refractive index, which modify the trajectory of the electromagnetic wave (thermal lensing effect) and, likewise, the optical field inside the cavity. According to Fermat's principle, light rays will follow a trajectory from point A to point $B$ that makes the optical path length stationary:

$$
\delta S=\delta \int_{A}^{B} n(\vec{r}) d s=0
$$

Where $n(\vec{r})$ represents the refractive index, $\vec{r}$ is the trajectory of the light and $s$ corresponds to the arclength parameter. The ray optics module of COMSOL solves the Hamiltonian equations derived from this variational principle [21]:

$$
\frac{d r_{i}}{d t}=\frac{\partial \omega}{\partial k_{i}} ; \frac{d k_{i}}{d t}=-\frac{\partial \omega}{\partial r_{i}}
$$

Where $k_{i}$ and $r_{i}$ are the components of the wavevector and ray trajectory, respectively, and $\omega$ the angular frequency.

To compute the effect of the temperature on the trajectory of the light, the dependence of the band gap for GaAs is calculated through Varshni's equation [22]

$$
E_{g}(T)=G_{g}(0)-\frac{\alpha T^{2}}{T+\beta}
$$

where $\mathrm{Eg}(0)=1.519 \mathrm{eV}$ is the band gap of GaAs at $0 \mathrm{~K}$, and the parameters $\alpha=5.40510^{-4} \mathrm{eV} / \mathrm{K}$ and $\beta=204 \mathrm{~K}$ for GaAs [23]. Once the band gap energy is known for each temperature the refractive index can be evaluated [24]. The temperature of the QW under operation is close to RT, while a local high temperature results in the shrinkage of the band gap by a value $\Delta E=E_{g}(300 \mathrm{~K})-E_{g}(T)$. This modifies the optical properties of the region under the influence of the local heating, as experimentally observed for the absorption coefficient [25,26], the refractive index [27], and the dielectric function [28]. The effect of the band gap reduction on the refractive index is basically equivalent to considering light propagating at the operational temperature of the device albeit with a higher energy: $\mathrm{E}(\mathrm{T})=h v^{\prime}=h v+\Delta \mathrm{E}$ (see Fig. 2). The energy of the laser radiation is approximately that of the room temperature band gap of GaAs, hv $=\mathrm{Eg}(300 \mathrm{~K})=1.42 \mathrm{eV}$. Hence, we arrive at: 


$$
E(T)=h v+\Delta E=2 E_{g}(300 K)-E_{g}(T)
$$

Finally, the experimental data for the refractive index of GaAs at $300 \mathrm{~K}$ as a function of the incident light energy $\mathrm{n}(\mathrm{E})$ can be obtained from [29], Figure 2.

The hot spot is modelled as a Gaussian temperature distribution:

$$
T(x, y)=T_{0}+\Delta T e^{-\left(\frac{x^{2}+y^{2}}{\sigma^{2}}\right)}
$$

The spot diameter is chosen to be of the order of $1 \mu \mathrm{m}(\sigma=1$ $\mu \mathrm{m})$. The maximum temperature of the distribution, $(\mathrm{TO}+\Delta \mathrm{T})$, is swept from room temperature $\left(\mathrm{T}_{0}=300 \mathrm{~K}, \Delta \mathrm{T}=0\right)$ up to the melting point for GaAs ( $1500 \mathrm{~K})$. Finally, this temperature distribution is substituted in Eq. 4, which leads to the expression for the refractive index as a function of the position, $\mathbf{n}(\mathbf{x}, \mathbf{y})$.

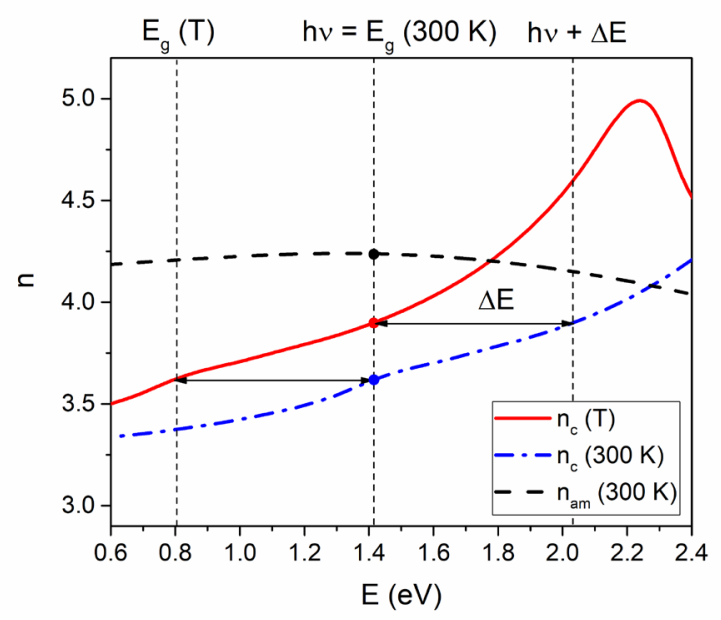

Fig. 2. Dependence of the refractive index of crystalline GaAs with the incident light energy at $300 \mathrm{~K} \quad[27,29]$ (dash-dot blue curve) and at a higher temperature $\mathrm{T}$ (full red curve). The figure also illustrates the equivalence of the indices $n(T, h v)=n(300 K, h v+\Delta E)$. The amorphous GaAs refractive index is also represented (dashed black curve).

The model has been solved with two different modules of COMSOL Multiphysics: Ray Optics and Electromagnetic Waves in Frequency Domain (EWFD). The first one directly solves Eqs. 2 to obtain the ray trajectories. The second one solves the complete set of Maxwell equations considering the phase variations of the EM field, which are critical for the study of nanoscale systems. The solution of the model provides the laser field distribution in the $\mathrm{QW}$ plane. The results of both approaches are equivalent as will be shown later on; however, the EWFD calculation should be regarded as a more accurate physical approach.

The other effect that will be scrutinised is the consequence of a degraded region on the distribution of the laser radiation. Once the mechanical limit of the material is overcome, its properties are locally deteriorated and it eventually stops emitting light, as it has been shown in Figure 1b. In extreme cases the material can locally reach its melting point, resulting in a subsequent recrystallization and change of the optical properties. We performed a simulation in which a damaged region with micrometric dimensions is placed inside the QW2 $\boldsymbol{\mu m}$ from the front facet of the device. In order to account for the drastically different optical properties of the degraded GaAs, the refractive index of that region was set to that of amorphous GaAs at $1.42 \mathrm{eV}(\mathrm{n}=4.2323$ and $\mathrm{k}=$ 0.8467) [30,31]. The model is solved by using the EWFD module to obtain the electromagnetic field distribution and to analyze the changes induced by the optical defect.

\section{Effect of high thermal gradients: thermal lensing effect}

The effect of high thermal gradients has been studied by both ray optics and wave optics approaches. The results derived from the simulations are equivalent and they are shown in Figures 3 and 4, respectively. In both cases the travelling plane wave is focused at a certain point behind the hot spot, showing a thermal lensing effect.

In the ray optics picture, Figure 3 , the focus progressively approaches the hot spot as its local temperature is increased, with a minimum distance of $5-6 \mu \mathrm{m}$ at temperatures approaching GaAs melting point. This scenario is in good agreement with the $\mathrm{CL}$ images of degraded devices, Figure $1 \mathrm{~b}$.

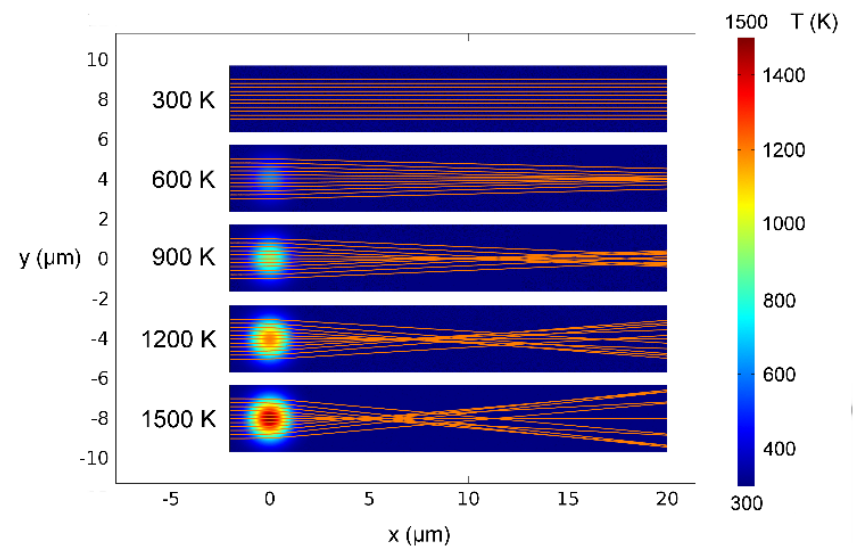

Fig. 3. Ray Optics simulation of the effect of a local heat spot over the laser beam trajectory. The laser beam is focused at a certain point beyond the thermal singularity. The distance between the focus and the heat spot decreases as the maximum temperature raises. Note that the presence of optical aberrations prevents a clear determination of a unique focal point, resulting in a slightly wide spot, instead of a perfect point-like focus. 


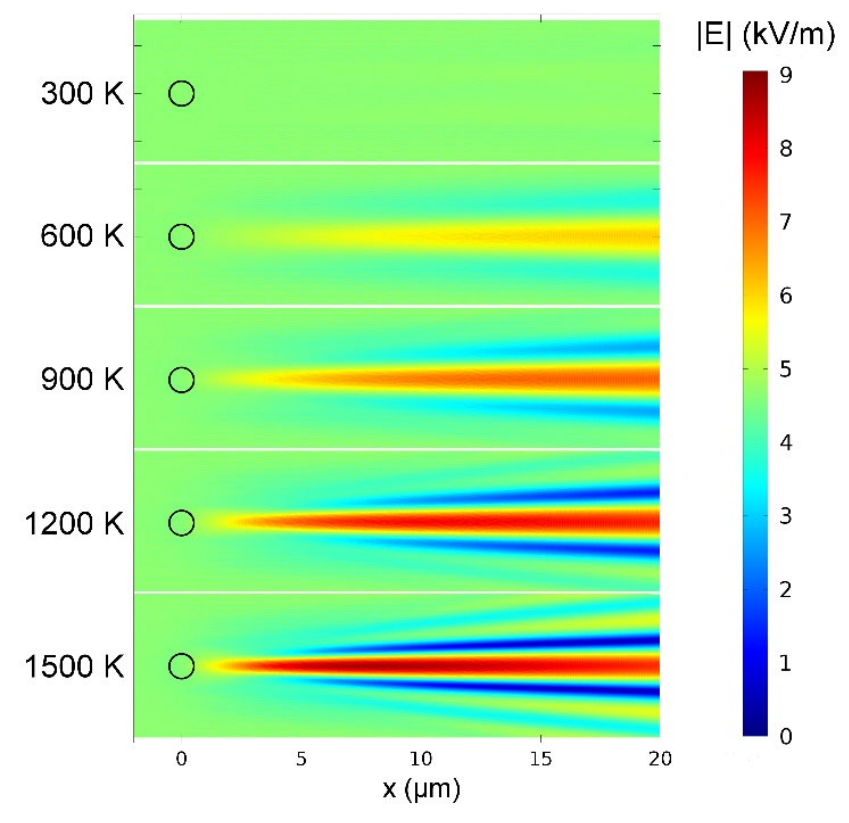

Fig. 4. Simulations with the Electromagnetic Waves in Frequency Domain module of COMSOL Multiphysics. The EM field is localized in a region past the heated region (black circle) that progressively approaches the defect as the maximum temperature raises. The results are equivalent to those of the ray optics approach, Figure 3.

The results obtained from the EWFD module, Figure 4, are much more consistent than the results of the ray optics solution. This was expected a priori, since the dimension of the heat spot is close to the nanometric range, being comparable to the laser wavelength. Thus, phase differences will play a significant role and physical optics should be used to obtain precise results. Figure 4 shows that the EM field is reinforced around the same region where the beam is focused in Figure 3. It can also be appreciated that the EM field is increasingly localised as the temperature of the hot spot raises.

\section{Impact of micrometric degraded regions on the laser field}

This second section deals with the effect of an already degraded region on the distribution of the laser electromagnetic field. The results are plotted in Figure 5. The first simulation corresponds to a $1 \mu \mathrm{m}$ wide damaged section that is spread $1 \mu \mathrm{m}$ along the cavity. The model shows an enhancement of the laser radiation in a segment that is extended $\sim 4 \mu \mathrm{m}$ further inside the $\mathrm{QW}$, Figure $5 \mathrm{~b}$. This is in good agreement with the experimental CL measurements and the thermal lensing simulations. As we have mentioned, both optical absorption and laser power density are critical issues of the degradation; therefore, the increased power density resulting from the thermal focusing a few microns beyond the initially overheated region will induce a new hot spot that would eventually lead to a new damaged region. In a recurrent way, each damaged section will induce a new hot spot, until lasing ends. We simulated this sequential process by extending the initial defect by steps of $4 \mu \mathrm{m}$ inside the $Q W$, Figures 5 b-e. As it can be observed, the optical field is concentrated behind the damaged region increasing the local laser intensity, promoting the appearance of a new degraded region. This effect, together with the thermal lensing mechanism, can account for the propagation of the DLDs along the laser cavity following the trends revealed by the CL images.

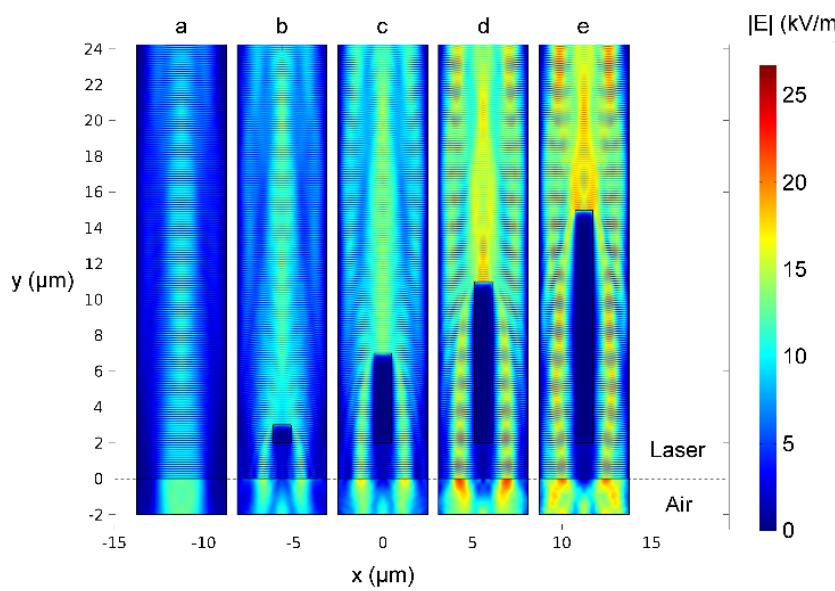

Fig. 5. Simulation of the electromagnetic field distribution of the laser radiation in the presence of a degraded region on the QW. The electromagnetic field is concentrated $\sim 4 \mu \mathrm{m}$ in front of the degraded region in all cases. This would eventually trigger a new local COD event, extending the damaged region. a) Field distribution in the absence of defects. b)-e) Field distributions in the presence of degraded regions with extensions $1,5,9$ and $13 \mu \mathrm{m}$, respectively.

It can also be noticed that the laser field is able to circumvent the damaged region. The local damage of the $Q W$ is unable to stop lasing operation, unless the length of the $Q W$ damaged region reaches a value for which the total gain is below the lasing threshold. Otherwise, when the damage extends to the guide layers the cavity is destroyed and lasing ends.

Finally, it is important to note that the possible changes on the local gain (e.g. spatial hole burning (SHB)) has not been considered in the model. Firstly, because this kind of effect tend to lower the gain locally, which would prevent the generation of new defects. Secondly, because the inhomogeneity induced by SHB in the carrier and light distributions present lengths $>100 \mu \mathrm{m}$ for long range SHB, and $<1 \mu \mathrm{m}$ for short range SHB (Ryvkin et al, J. Appl. Phys 109, 043101 (2011)). In both cases, the SHB intensity patterns are different from the pattern generated by thermal lensing

\section{Conclusions}

The effect of thermal and structural singularities in the QW on the COD processes of high-power laser diodes have been studied. First, the impact of high thermal gradients around a local hot spot on the laser beam trajectory has been analysed. A 2D model with a $1 \mu \mathrm{m}$ Gaussian temperature profile has been solved by using both rays and wave optics. The results of both approaches are equivalent, the wave optics solution being more consistent, but the ray solution displays a very intuitive image of the thermal lensing phenomenon. In both 
cases the travelling wave is focused by thermal lensing on a region situated $4-6 \mu \mathrm{m}$ behind the hot spot. Second, we investigated the effect of a fully degraded region of material within the QW. A $1 \mu \mathrm{m} 2$ region of amorphous GaAs located near the front facet of the laser has been modelled. The results show the preferential localization of the electromagnetic field $\sim 4 \mu \mathrm{m}$ behind the degraded region. This field localization, possibly combined with the thermal lensing effect, could trigger the sequential occurrence of a new COD event at the concentration focus, in a similar way to what is observed on CL measurements. The degraded region is then extended according to the degradation of the new hot spot and the simulation is repeated. The electromagnetic field is again localized at a new point located $\sim 4 \mu \mathrm{m}$ behind the new extended defect. Therefore, the degradation of the $Q W$ can occur sequentially according to this scheme.

As a summary, the results of both approaches show a concentration of the laser beam close behind the heated or degraded area inside the $\mathrm{QW}$. This laser beam concentration could trigger new sequential COD events, explaining the "chain" of dark spots observed in high power laser diodes presenting COD failure.

Funding. Junta de Castilla y León (VA283P18), Spanish MINECO (ENE 2014-56069-C4-4-R), Spanish Government (FPU14/00916).

Disclosures. The authors declare no conflicts of interest. 


\section{References}

1. J. Neukum, "Innovation enriches high power diode laser market," Opt. Laser Eur. 165, 37-39 (2008).

2. J. W. Tomm and J. Jiménez, Quantum-Well Laser Array Packaging (McGraw-Hill, 2007).

3. J. W. Tomm, M. Ziegler, M. Hempel, and T. Elsaesser, "Mechanisms and fast kinetics of the catastrophic optical damage (COD) in GaAs-based diode lasers," Laser Photon. Rev. 5, 422-441 (2011).

$4 . \quad$ J. Souto, J. L. Pura, and J. Jiménez, "Thermomechanical issues of high power laser diode catastrophic optical damage," J. Phys. D. Appl. Phys. 52, 343002 (2019).

5. B. W. Hakki and F. R. Nash, "Catastrophic failure in GaAs double-heterostructure injection lasers," J. Appl. Phys. 45, 3907-3912 (1974).

6. C. H. Henry, P. M. Petroff, R. a. Logan, and F. R. Merritt, "Catastrophic damage of AlxGa1-xAs doubleheterostructure laser material," J. Appl. Phys. 50, 37213732 (1979).

7. Y. Sin, N. Presser, B. Foran, and S. C. Moss, "Catastrophic Facet and Bulk Degradation in High Power Multi-Mode InGaAs Strained Quantum Well Single Emitters Yongkun," Proc. SPIE 7198, 719818-1-719818-12 (2009).

8. M. Hempel, J. W. Tomm, V. Hortelano, N. Michel, J. Jiménez, M. Krakowski, and T. Elsaesser, "Time-resolved reconstruction of defect creation sequences in diode lasers," Laser Photon. Rev. 6, L15-L19 (2012).

$9 . \quad$ J. Souto, J. L. Pura, and J. Jiménez, "Thermal and mechanical issues of high-power laser diode degradation," MRS Commun. 1-5 (2018).

10. P. E. Hopkins, J. C. Duda, S. P. Clark, C. P. Hains, T. J. Rotter, L. M. Phinney, and G. Balakrishnan, "Effect of dislocation density on thermal boundary conductance across GaSb/GaAs interfaces," Appl. Phys. Lett. 98, (2011).

11. P. G. Eliseev, "Degradation of injection lasers," J. luminiscence 7, 338-356 (1973).

12. M. Hempel, J. W. Tomm, M. Ziegler, T. Elsaesser, N. Michel, and M. Krakowski, "Catastrophic optical damage at front and rear facets of diode lasers," Appl. Phys. Lett. 97, 10-13 (2010).

13. A. Martín-Martín, M. Avella, M. P. Iñiguez, J. Jiménez, M. Oudart, and J. Nagle, "A physical model for the rapid degradation of semiconductor laser diodes," Appl. Phys. Lett. 93, 171106 (2008).

14. A. Martín-Martín, M. Avella, M. P. Iñiguez, J. Jiménez, M. Oudart, and J. Nagle, "Thermomechanical model for the plastic deformation in high power laser diodes during operation," J. Appl. Phys. 106, 073105 (2009).

15. A. Martín-Martín, P. Iñiguez, J. Jiménez, M. Oudart, and J. Nagle, "Role of the thermal boundary resistance of the quantum well interfaces on the degradation of high power laser diodes," J. Appl. Phys. 110, 033113 (2011).

16. J. Souto, J. L. Pura, and J. Jiménez, "About the physical meaning of the critical temperature for catastrophic optical damage in high power quantum well laser diodes," Laser Phys. Lett. 13, 025005 (2016).

17. J. Souto, J. L. Pura, A. Torres, J. Jiménez, M. Bettiati, and F. J. Laruelle, "Sequential description of the catastrophic optical damage of high power laser diodes," in Proc. SPIE, M. S. Zediker, ed. (2016), Vol. 9733, p. 973306.

18. J. Souto, J. L. Pura, and J. Jiménez, "Nanoscale effects on the thermal and mechanical properties of AlGaAs/GaAs quantum well laser diodes: Influence on the catastrophic optical damage," J. Phys. D. Appl. Phys. 50, 235101 (12pp) (2017).

19. J. Souto, J. L. Pura, A. Torres, J. Jiménez, M. Bettiati, and F. J.
Laruelle, "Catastrophic optical damage of high power InGaAs/AlGaAs laser diodes," Microelectron. Reliab. 64, 627-630 (2016).

$20 . \quad$ J. Souto, J. L. L. Pura, A. Torres, and J. Jiménez, "Thermomechanical degradation of single and multiple quantum well AlGaAs/GaAs laser diodes," Microelectron. Reliab. 76-77, 588-591 (2017).

21. Ray Optics Module User's Guide. COMSOL Multiphysics $®$ v. 4.3a. COMSOL AB, Stockholm, Sweden (2008).

22. Y. P. Varshni, "Temperature dependence of the energy gap in semiconductors," Physica (1967).

23. J. S. Blakemore, "Semiconducting and other major properties of gallium arsenide," J. Appl. Phys. 53, (1982).

24. P. Geng, W. Li, X. Zhang, Y. Deng, H. Kou, L. Chen, and X. $\mathrm{Wu}$, "Effects of temperature and redshift on the refractive index of semiconductors," J. Appl. Phys. 124, 035703 (2018).

$25 . \quad$ M. D. Sturge, "Optical absorption of gallium arsenide between 0.6 and 2.75 eV," Phys. Rev. 127, 768-773 (1962).

26. D. D. Sell and H. C. Casey, "Optical absorption and photoluminescence studies of thin GaAs layers in GaAsSingle Bond signAlxGa1-xAs double heterostructures," J. Appl. Phys. 45, 800-807 (1974).

$27 . \quad$ D. T. F. Marple, "Refractive index of GaAs," J. Appl. Phys. 35, 1241-1242 (1964).

28. H. Yao, P. G. Snyder, and J. A. Woollam, "Temperature dependence of optical properties of GaAs," J. Appl. Phys. 70, 3261-3267 (1991).

29. S. Adachi, "Optical dispersion relations for $\mathrm{GaP}, \mathrm{GaAs}$, GaSb, InP, InAs, InSb, Al x Ga 1-x As, and In 1-x Ga x As y P 1-y," J. Appl. Phys. 66, 6030-6040 (1989).

30 S. Adachi, "Optical properties of gaas partially amorphized by ion implantation: Effective-medium-approximation analysis," J. Appl. Phys. 69, 7768-7773 (1991).

31. A. Gheorghiu and M. L. Thèye, "Disorder effects and the optical properties of amorphous GaAs and GaP," Philos. Mag. B Phys. Condens. Matter; Stat. Mech. Electron. Opt. Magn. Prop. 44, 285-306 (1981). 